\title{
Is a polygenic predictor of antidepressant response a possibility?
}

\author{
"...the genetic component of antidepressant response was \\ demonstrated to be highly polygenic and largely explained (42\% of \\ individual differences) by common polymorphisms throughout the \\ whole genome."
}

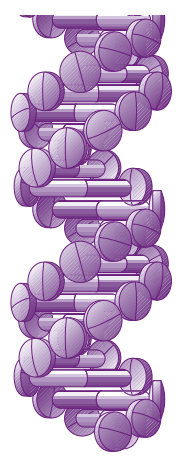

First draft submitted: 24 March 2017; Accepted for publication: 6 April 2017; Published online: 8 June 2017

Keywords: GWAS • methodology • psychiatric PGx

The hypothesis that antidepressant response has a relevant genetic component was initially suggested by the observation that this phenotype clusters in families [1]. Since the first pharmacogenetic studies investigated antidepressant efficacy in the 1990s, methodological innovations have been outstanding from a qualitative and quantitative point of view, moving from the analysis of few polymorphisms to millions and from statistics focused on single variants to multimarker tests. Candidate gene studies have been focused on a limited number of polymorphisms in genes that were thought to play a pivotal role in antidepressant mechanisms of action. The selection of candidate genes was usually based on data from preclinical studies, thus the risk of false positive findings was reduced by increasing the pretest probability, but the limitations of this approach became evident as antidepressant response proved to be a highly polygenic trait [2].

Candidate gene studies were a way to refine findings and provide small pieces of knowledge in the context of a very complex puzzle. Indeed, the genetic component of antidepressant response was demonstrated to be highly polygenic and largely explained ( $42 \%$ of individual differences) by common polymorphisms throughout the whole genome [3]. The strongest candidate genes were not free from negative or contradictory findings, and the most recent and comprehensive meta-analysis found that their effect sizes were modest at best (odds ratio usually around 1.3-1.5) [4].

Genome-wide association studies (GWAS) were also unable to provide revolutionary findings in the field, despite the fact they started from the premise of providing the basis for discovering the multiple variants contributing to antidepressant response. Current GWAS cover millions of common variants within the whole genome, but few polymorphisms reached the genome-wide significant threshold and they were not replicated across different samples [2]. Since convergent data demonstrated that genetic variants contribute to a relevant percentage of variance in antidepressant response, the consequent conclusion is that we should still look for the suitable methodology to find them. The starting point may be to consider the main GWAS weaknesses: GWAS per se do not explain the polygenic nature of antidepressant response if the focus is on genomewide significant hits; polymorphisms with genuine but small effect size may not reach the genome-wide significant threshold in samples of hundreds to a few thousands subjects; it is difficult to understand which nongenome-wide significant findings may be of some value because their biological role is often unknown. Taking into account these issues, a couple of critical points to address are: to use a methodology to account

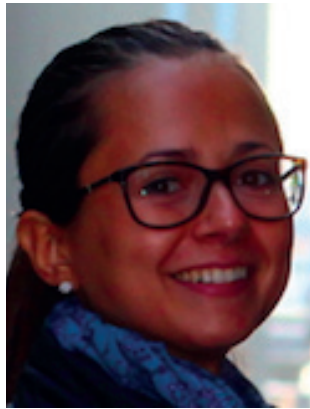

Chiara Fabbri

Department of Biomedical \& Neuromotor Sciences, University of Bologna, Viale Carlo Pepoli 5, 40123 Bologna, Italy and

Institute of Psychiatry, Psychology \& Neuroscience, King's College London, UK

Tel.: +390516584233 chiara.fabbri@yahoo.it 
for the effect of multiple variants and to improve the understanding of the biological mechanisms involved.

\footnotetext{
"The relatively recent origin of polygenic risk factors as an analytic approach to complex traits and the results obtained for other traits suggest that methodological improvements are needed to exploit the potential of polygenic risk scores in identifying the genetics of antidepressant response.
}

Polygenic risk scores (PRS) are a recent analysis approach that try to address the first point, but it could also be useful to provide a better knowledge of the molecular pathways mediating antidepressant action. PRS aim to predict the polygenic component of the phenotype using a flexible sum of the effect sizes of associated polymorphisms. Flexibility is conferred by the fact that typically polygenic scores are calculated at different $\mathrm{p}$ thresholds, from a small number (e.g., starting from a given value and adding 0.05 or 0.10 up to 1 ) to thousands (with small increments from the lower to the upper bound, e.g., increments of 0.00005). The use of this flexibility is a way to face the problem of avoiding the exclusion of polymorphisms with small but potentially genuine association or association per se not significant but relevant in conjunction with the effect of other variants. Thus, a higher level of uncertainty is allowed compared with GWAS in order to improve prediction, but multiple-testing correction should always be applied when taking into account the different $\mathrm{p}$-thresholds tested. The most used approach in existing studies consists of calculating the PRS as the sum of associated alleles, weighted by effect sizes, for polymorphisms with p-values less than predefined thresholds in a training sample, then to test the predictive ability of the PRS in a replication or target sample. Polymorphisms are usually clumped by linkage disequilibrium and $p$-value to avoid result overfitting: the polymorphism with the smallest $\mathrm{p}$-value within a given window is retained, and all SNPs in linkage disequilibrium with the retained polymorphism are excluded [5]. PRS were applied for predicting antidepressant efficacy in major depressive disorder (MDD) although few studies have been carried out to date [6-8]. Two of these studies used individual GWAS as training samples $[7,8]$ while the other calculated the PRS in a meta-analysis of two GWAS (training sample) [6]. The latter study reported that the PRS was significantly associated with symptom improvement and remission in the target sample, and the strongest prediction was achieved with the threshold of $\mathrm{p}<0.05$. For both outcomes, the PRS included approximately 15,000 independent markers and explained between 0.5 and $1.2 \%$ of variance in outcomes in the target sample. The other two studies did not find a significant predictive effect of the PRS in the targeted GWAS, and the exclusion of subjects not treated with selective serotonin reuptake inhibitors did not improve the results $[7,8]$.

Several factors are probably involved in the failure/unsatisfying findings of using PRS to create a reliable predictor of antidepressant efficacy. The most macroscopic one is the evidence of lack of adequate statistical power in samples used to estimate PRS (from $~ 800$ to 1400 subjects): it was estimated that a sample size ten-times larger would be required to achieve $80 \%$ power for polygenic prediction between studies of antidepressant response [8]. The use of the results of a GWAS meta-analysis for calculating the PRS is expected to improve the power by increasing sample size and including polymorphisms that show a consistent association signal in different samples. The recruitment of samples providing adequate power is, however, not easy to solve, since not only ascertainment of diagnosis but also the characterization of antidepressant response is necessary. National registers and electronic medical records of large healthcare organizations could be used to achieve a study of this magnitude, but substantial resources would also be needed. In order to make such an ambitious study possible, self-reported measures of diagnosis and antidepressant use history have been collected in the 23andMe cohort, which includes approximately 48,000 subjects and which was used to perform a GWAS investigating antidepressant outcomes but not a PRS study [9]. A framework to compare the genetic contribution to MDD and self-reported depression was recently proposed. Higher heritability and higher contribution of rare variants was reported for self-reported depression compared with MDD, thus it was possible that the former included subjects with other psychiatric diagnosis and the reliability of self-diagnosed depression remains not completely clear [10].

In addition to increasing sample size, other methodological strategies could be applied in order to improve the power of PRS in detecting the genetic component of antidepressant response. These strategies include the use of variant annotation to prioritize polymorphisms and other corrections such as winner's curse adjustments [11]. Variant annotation can take into account different types of information, in particular: location in a coding region, functional role and level of conservation of the variant [12]; expression and methylation quantitative trait loci; results of existing GWAS at SNP, gene and pathway level. The application of a less rigorous $p$ threshold for inclusion of polymorphisms with higher pretest probability of involvement in the phenotype (e.g., functional variants or variants in conserved genomic regions) was demonstrated to improve the prediction of PRS 
for a number of different traits [11]. Another idea is to calculate PRS including only variants in pathways that have been associated with the phenotype or prioritize these variants. Pathway-level regional heritability was demonstrated to be significant in MDD and a PRS calculated including variants from the Netrin-1 signaling pathway showed competitive predictive accuracy compared with the whole-genome PRS [13]. PRS applied to specific pathways may also contribute to the clarification of mechanisms of antidepressant action. A possible approach that could be used in combination with the previous ones is the identification of specific groups of patients on the basis of clinical information, with the aim of reducing the heterogeneity of the genetic component involved in antidepressant response. The genetics of MDD is very heterogeneous compared with other psychiatric disorders as SNP correlation was demonstrated to be slightly above $50 \%$ between independent MDD samples and over $80 \%$ between independent schizophrenia samples [14]. Consistently, the partitioning of MDD in different clinical subtypes such as typical and atypical MDD was demonstrated to improve the predictive performance of PRS [15] and a similar approach could be useful for antidepressant efficacy. Different MDD subtypes were demonstrated to have different outcomes of antidepressant treatment, for example, chronic MDD or anxious MDD were demonstrated to be particularly at risk of treatment failure [16]. Another point to consider is that depressive symptoms are not all the same, but different dimensions were identified. For example, anhedonia is a dimension that predicts a negative outcome of treatment [17] and 44\% of variance in this phenotype was attributed to genetic factors [18]. The use of specific symptom dimensions has been successfully applied in PRS studies investigating schizophrenia and bipolar disorder [19].

A further interesting hypothesis is that the risk conferred by PRS may be affected by environmental exposures, particularly stressful life events. A previous study demonstrated that PRS and stressful life events had an additive effect on the risk of developing depressive symptoms [20], suggesting another approach that could also be applied to the study of antidepressant response.

In conclusion, available studies have provided weak evidence supporting the usefulness of PRS to predict antidepressant efficacy so far. The relatively recent origin of PRS as an analytic approach to complex traits and the results obtained for other traits suggest that methodological improvements are needed to exploit the potential of PRS in identifying the genetics of antidepressant response. Until the recruitment of samples providing adequate power is achieved, other strategies have been proposed in order to optimize the power of existing samples, in particular SNP prioritization based on annotation, other corrections such as winner's curse adjustments [11], analysis of clinically more homogenous groups of patients or specific symptom dimensions and study of the additive effect between PRS and stressful life events. A successful application of PRS to the prediction of antidepressant response may provide pharmacogenomic guidance to antidepressant treatment in the future, allowing the classification of patients within a spectrum of risk for specific phenotypes such as treatment-resistance.

\section{Financial \& competing interests disclosure}

The author has no relevant affiliations or financial involvement with any organization or entity with a financial interest in or financial conflict with the subject matter or materials discussed in the manuscript. This includes employment, consultancies, honoraria, stock ownership or options, expert testimony, grants or patents received or pending, or royalties.

No writing assistance was utilized in the production of this manuscript.

\section{References}

1 Franchini L, Serretti A, Gasperini M, Smeraldi E. Familial concordance of fluvoxamine response as a tool for differentiating mood disorder pedigrees. J. Psychiatr. Res. 32(5), 255-259 (1998).

2 Fabbri C, Crisafulli C, Calabrò M, Spina E, Serretti A. Progress and prospects in pharmacogenetics of antidepressant drugs. Expert Opin. Drug Metab. Toxicol. 27, 1-12 (2016).

3 Tansey KE, Guipponi M, Hu X et al. Contribution of common genetic variants to antidepressant response. Biol. Psychiatry 73(7), 679-682 (2013).

4 Niitsu T, Fabbri C, Bentini F, Serretti A. Pharmacogenetics in major depression: a comprehensive

meta-analysis. Prog. Neuropsychopharmacol. Biol. Psychiatry 45, 183-194 (2013).

5 Euesden J, Lewis CM, O'Reilly PF. PRSice: polygenic risk score software. Bioinformatics 31(9), 1466-1468 (2015).

6 GENDEP Investigators, MARS Investigators, STAR*D Investigators. Common genetic variation and antidepressant efficacy in major depressive disorder: a meta-analysis of three genome-wide pharmacogenetic studies. Am. J. Psychiatry 170(2), 207-217 (2013).

7 Tansey KE, Guipponi M, Perroud N et al. Genetic predictors of response to serotonergic and noradrenergic antidepressants in major depressive disorder: a genome-wide analysis of individuallevel data and a meta-analysis. PLoS Med. 9(10), e1001326 (2012). 
8 García-González J, Tansey KE, Hauser J et al. Pharmacogenetics of antidepressant response: a polygenic approach. Prog. Neuropsychopharmacol. Biol. Psychiatry 75, 128-134 (2017).

9 Li QS, Tian C, Seabrook GR, Drevets WC, Narayan VA. Analysis of 23andMe antidepressant efficacy survey data: implication of circadian rhythm and neuroplasticity in bupropion response. Transl. Psychiatry 6(9), e889 (2016).

10 Fabbri C. Genetic and environmental contribution to major depressive disorder and self-declared depression. EBioMedicine 14, 7-8 (2016).

11 Shi J, Park J-H, Duan J et al. Winner's curse correction and variable thresholding improve performance of polygenic risk modeling based on genome-wide association study summary-level data. PLoS Genet. 12(12), e1006493 (2016).

12 Finucane HK, Bulik-Sullivan B, Gusev A et al. Partitioning heritability by functional annotation using genomewide association summary statistics. Nat. Genet. 47(11), 1228-1235 (2015).

13 Zeng Y, Navarro P, Fernandez-Pujals AM et al. A combined pathway and regional heritability analysis indicates Netrin 1 pathway is associated with major depressive disorder. Biol. Psychiatry 81(4), 336-346 (2016).
14 Gratten J, Wray NR, Keller MC, Visscher PM. Large-scale genomics unveils the genetic architecture of psychiatric disorders. Nat. Neurosci. 17(6), 782-790 (2014).

15 Milaneschi Y, Lamers F, Peyrot WJ et al. Polygenic dissection of major depression clinical heterogeneity. Mol. Psychiatry 21(4), 516-522 (2016).

16 De Carlo V, Calati R, Serretti A. Socio-demographic and clinical predictors of non-response/non-remission in treatment resistant depressed patients: a systematic review. Psychiatry Res. 240, 421-430 (2016).

17 McMakin DL, Olino TM, Porta G et al. Anhedonia predicts poorer recovery among youth with selective serotonin reuptake inhibitor treatment-resistant depression. J. Am. Acad. Child Adolesc. Psychiatry 51(4), 404-411 (2012).

18 Bogdan R, Pizzagalli DA. The heritability of hedonic capacity and perceived stress: a twin study evaluation of candidate depressive phenotypes. Psychol. Med. 39(2), 211-218 (2009).

19 Ruderfer DM, Fanous AH, Ripke S et al. Polygenic dissection of diagnosis and clinical dimensions of bipolar disorder and schizophrenia. Mol. Psychiatry 19(9), 1017-1024 (2014).

20 Musliner KL, Seifuddin F, Judy JA, Pirooznia M, Goes FS, Zandi PP. Polygenic risk, stressful life events and depressive symptoms in older adults: a polygenic score analysis. Psychol. Med. 45(8), 1709-1720 (2015). 